\title{
Linx
}

Revue des linguistes de l'université Paris X Nanterre

$54 \mid 2006$

La cause : approche pluridisciplinaire

\section{Étude de GP en dans, à sens causal}

Céline Vaguer

\section{OpenEdition}

\section{Journals}

Édition électronique

URL : http://journals.openedition.org/linx/506

DOI : $10.4000 /$ linx.506

ISSN : 2118-9692

\section{Éditeur}

Presses universitaires de Paris Nanterre

\section{Édition imprimée}

Date de publication : 1 juin 2006

Pagination : 91-102

ISSN : 0246-8743

\section{Référence électronique}

Céline Vaguer, «Étude de GP en dans, à sens causal », Linx [En ligne], 54 | 2006, mis en ligne le 01 août 2007, consulté le 19 avril 2019. URL : http://journals.openedition.org/linx/506 ; DOI : 10.4000/linx.506 


\section{Étude de GP en dans, à sens causal}

\section{Céline Vaguer, Université Paris X-Nanterre, MoDyCo (UMR 7114)}

A qui se préoccupe de saisir le fonctionnement du système linguistique lui-même, il paraît plus cohérent de chercher à caractériser le sens d'une préposition à l'aide des relations syntaxiques et distributionnelles qu'elle tisse avec son cotexte ${ }^{1}$ que de partir d'un postulat selon lequel le sens spatial présiderait à l'ensemble des emplois ${ }^{2}$. Qui plus est, on ne peut pas " partir» de la notion sémantique utilisée pour définir la préposition, étant donné que cette notion est susceptible d'en regrouper plusieurs ainsi la notion de «direction» concerne les prépositions (à, pour, vers, sur...), celle d'« approximation » est utilisable pour vers, dans (Il pèse dans les 100 kilos) et sur (Il habite sur Bordeaux), celle de «cause » vaut pour les prépositions de, par, pour - et que la notion change selon l'emploi de la préposition (dans n'est plus spatial dans des énoncés comme Il pleuvra dans deux jours et vers peut indiquer la direction ou l'approximation : $V$ enez vers midi). Ranger les prépositions en classes notionnelles reviendrait à multiplier

\footnotetext{
${ }^{1}$ Environnement linguistique (syntaxique et distributionnel) de la phrase analysée.

${ }^{2}$ Les linguistes cognitivistes privilégient l'expression de l'espace (cf. en particulier C. Vandeloise (1986) ou G. Lakoff (1987) dont l'objectif est de décrire les prépositions spatiales), rompant avec la tradition structuraliste qui s'efforçait de construire un signifié (en langue) susceptible de subsumer tous les effets observables en discours (G. Guillaume 1919, V. Brøndal 1950, B. Pottier 1955, par exemple). Il ne s'agit plus alors de saisir le fonctionnement d'un système posé comme autonome relativement à la réalité qui nous entoure ou à la manière dont nous nous la représentons, mais au contraire, à partir d'une hypothèse sur les mécanismes mentaux, d'établir un lien entre eux et le sens des unités linguistiques. Le postulat qui préside aux descriptions cognitivistes est qu'il y a un sens premier (jugé prototypique) d'ordre "concret" à la base de tous les autres emplois. Ainsi les prépositions sont-elles définies à partir de leur sens spatial (le plus concret) : la thèse est sur le bureau, jugé « prototypique» (c'est-à-dire fondamental, représentatif), les autres emplois en étant dérivés: veiller sur les enfants, avoir de l'influence sur quelqu'un...
} 
les homonymes avec, par exemple, un pour relevant de la classe des prépositions spatiales (partir pour Lyon), un autre de la classe des prépositions temporelles (partir pour deux jours), un autre des causales (avoir un congé pour maladie), etc.

L'hypothèse ici retenue est que l'analyse formelle (distributionnelle et syntaxique) permet de progresser dans l'investigation sémantique et que, réciproquement, ses résultats sur le plan du sens conduisent à des avancées en matière d'analyse grammaticale. Ainsi, si l'intuition première affecte aux groupes prépositionnels (GP) en dans une interprétation spatiale (Il y a une boulangerie dans la rue voisine, Hervé babite dans un appartement de $250 \mathrm{~m}^{2} \ldots$ ), une étude syntaxique permet de révéler que souvent en réalité s'en cache une autre, celle de causalité : l'énoncé se prête alors à une paraphrase de type c'est parce que $X$ (Elle a un congé pour maladie: c'est "parce qu'elle est malade qu'elle a un congé ». Il m'a pardonné dans sa magnanimité : «il m'a pardonné parce qu'il est magnanime $»$ ).

La méthodologie générale adoptée pour rendre compte du fonctionnement linguistique part du principe que l'on ne peut le faire qu'à partir d'un travail sur les formes (observation et découverte de leurs propriétés par l'étude des distributions et transformations qu'elles permettent ou excluent) et que ce que révèle l'analyse des formes (interprétation des compatibilités et incompatibilités ainsi dégagées) permet d'accéder au sens. Le sens est donc indissociable de la forme.

Mais on ne peut interpréter les prépositions en se limitant à cela. Il faut en effet trouver un complément d'information dans le cotexte. Le sens de la préposition est conditionné par l'étude simultanée du lexique, de la syntaxe, voire de la situation d'énonciation : ainsi par exemple le repère temporel dans un mois de Léa arrive dans un mois s'interprète relativement au moment où la phrase est énoncée. De même, l'étude lexicale permet de mettre en évidence que l'on ne peut se fier à la structure de surface comme l'illustrent les énoncés (1) et (2) : générosité relève du paradigme des noms de qualité et affolement de celui des noms d'état (plus précisément de sentiment) ${ }^{3}$. Par conséquent, leur composition interne n'est pas identique. De fait, sémantiquement, le GP en (1) a une valeur uniquement causale alors qu'en (2) on peut lui associer une valeur de 'cause-durée' (Leeman 1985). Ce sont ces observations qu'il nous faut généraliser afin de pouvoir caractériser ces GP en dans à valeur causale et le rôle attribué à la préposition car, à elle seule, la structure ne permet pas d'annoncer de manière intuitive la fonction que dans occupe au sein de la phrase.

(1) Dans leur générosité, Max et Léa ont laissé tout l'héritage à Tom.

(2) Dans leur affolement, Max et Léa ont oublié la présence de Marie.

\footnotetext{
${ }^{3}$ Ces étiquettes sémantiques ('qualité', 'état', 'sentiment') correspondent à des propriétés distributionnelles et syntaxiques ( $c$. Anscombre 1995, Flaux et Van de Velde 2000, Leeman 1995, entre autres) de même que 'action' ou 'événement » qui seront utilisés plus loin.
} 


\section{Mise en évidence de la valeur causale : la structure interne du GP}

\subsection{La préposition}

Bien que la plupart des prépositions soient polysémiques, il en existe qui apparaissent - au moins à en croire les descriptions lexicographiques - monosémiques, comme malgré, qui semble n'avoir trait qu'à l'opposition. En particulier, les locutions à cause de, du fait de ou en raison de sont considérées comme susceptibles de n'exprimer que la cause et au cours de ou lors de seulement le temps : on partira de ces commutations - à équivalence sémantique globale - pour conclure à un emploi causal ou temporel de dans.

\subsubsection{Emploi de dans à valeur causale}

(a) Dans est commutable avec à cause de lorsque la proposition dans laquelle prend place le GP en dans réfère à un fait susceptible d'être interprété négativement :

(3) (Dans + A cause de) l'ablation d'un sein, la plastique de la femme est modifiée : la plastique de la femme est modifiée parce qu'il y a eu l'ablation d'un sein.

(4) (Dans $+A$ cause de) l'explosion, des toits ont été arrachés.

(5) Il m'inquiète (dans + à cause de) ses manifestations d'bumeur.

(6) (Dans $+A$ cause du) choc, la moto a perdu sa roue.

(7) (Dans $+A$ cause de) son agitation, le malade renversa le verre posé sur la table de nuit.

(b) Dans est commutable avec des prépositions du type grâce à lorsque le procès est compris comme renvoyant à quelque chose que l'on peut juger positivement :

(8) (Dans + Grâce à) la fouille, d'intéressants ossements ont été mis au jour : c'est parce qu'il y a eu la fouille que les ossements ont été découverts.

(9) La nouvelle entité se spécialiserait (dans + grâce à) la gestion de fonds de pension.

(10) La vie se perpétuait (dans les + grâce aux) étables.

(11) Les prélèvements d'eau sont réalisés (dans les + grâce aux) bassins.

(12) (Dans + Grâce à) ses alluvions, le géologue décela du calcaire.

(c) Du fait de commute avec dans, plus facilement que grâce à et à cause de, dans certaines situations qui ne seraient pas, de par leur nature même, provocatrices d'action pouvant être jugées comme répréhensibles ou non :

(13) (Dans + Du fait de) son inspiration, ce texte nous fait accéder aux émotions de l'auteur.

(14) (Dans + Du fait de) leur générosité, Max et Léa ont laissé tout l'béritage à Tom.

(15) (Dans + Du fait de) son profond abattement, Max n'avait plus goût à rien.

(16) (Dans + Du fait de) son aburissement, Paul oublia de refermer la bouche.

(17) (Dans + Du fait de) sa bonté, Dieu créa une femme pour Adam. 
L'analyse du corpus ${ }^{4}$ montre que la substitution de dans à l'une ou l'autre des prépositions causales ne se fait pas de façon aléatoire : dans 35,24\% de nos énoncés à cause de se substitue naturellement à dans, dans 12,38 \% ce sera à grâce à et dans 52,38 \% des énoncés $d u$ fait de est à privilégier. Ces résultats sont en fait à corroborer au type de noms que l'on trouve dans le GP: en effet, à cause de entre en combinaison plus naturellement avec des $\mathrm{N}_{\text {action }}$ ou des $\mathrm{N}_{\text {événement, }}$ du fait de avec des $\mathrm{N}_{\text {état psychologique, et }}$

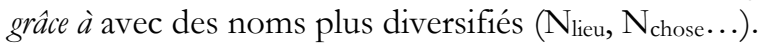

\subsubsection{Emploi de dans à valeur temporelle}

Parmi les GP ayant une valeur causale, certains peuvent se voir associer une valeur de durée. On sait que pendant indique une simultanéité continue entre $a$ et $b$ dans $a$ pendant $b$ lorsque $b$ est un nom de mesure du temps quantifié : Nous avons eu des congés pendant trois jours. Dans ce cas, on ne peut pas substituer dans à pendant: *Nous avons eu des congés dans trois jours. Mais cette simultanéité peut n'avoir lieu qu’à un moment donné de $b$ : Il a été malade pendant la nuit. Ici, dans est susceptible de commuter avec pendant: Il a été malade dans la nuit, comme au cours de: Il a été malade au cours de la nuit.

(18) (Dans + pendant + au cours de) son altercation avec Lia, Max s'est mis à pleurer.

(19) (Dans + pendant + au cours de + au moment de) l'ablation d'un sein, la plastique de la fermme est modifiée.

(20) Elle n'avait cessé de s'abêtir (dans + au cours d') une profonde absence.

(21) Le château a été détruit (dans + pendant + au cours de) la bataille.

(22) (Dans + au cours de) son abêtissement, Paul n'eut pas le courage de fermer la télé.

La préposition pendant est elle-même susceptible de deux commutations, avec au cours de et au moment de. Pendant est substituable à au cours de lorsque le nom introduit est à interpréter comme duratif ${ }^{5}$, c'est-à-dire désigne un procès pris comme point de repère qui est déjà en cours et peut être prolongé au-delà (rien n'est dit de son début ni de son éventuel achèvement: il est présenté sous l'aspect non limitatif). Ces noms permettent des distributions telles que :

La fouille commença le 4 novembre, continua pendant deux mois et se termina en janvier.

Pendant est aussi substituable à au moment de lorsque les actions sont ponctuelles : la durée du procès est alors entièrement comprise dans le nom, le procès est donc présenté sous l'aspect limitatif. Le nom est dans ce cas compatible avec des distributions telles que se passe, se produit, a lieu, survient (à un moment donné):

L'agression s'est produite à $10 \mathrm{~h}$ du matin, en pleine rue.

\footnotetext{
${ }^{4}$ Notre corpus englobe à la fois des exemples simplifiés à partir d'attestations relevées dans Frantext entre autres et des exemples empruntés aux travaux, principalement sur la cause, cités en bibliographie.

${ }^{5}$ Exemple de $\mathrm{N}_{\text {duratif }}$ : bataille, altercation, action, joute, bagarre, escalade, manutention...

Exemple de $\mathrm{N}_{\text {ponctuel }}$ : abaissement, abattage, abordage, agression, attentat, prouesses, erreurs...
} 
Certains noms peuvent avoir les deux interprétations suivant le sens que l'on attribue à l'énoncé. Ainsi ablation prend l'aspect duratif lorsqu'il est entendu comme une opération et l'aspect ponctuel lorsque l'on pense à l'action même, au moment même de l'extraction.

Le corpus comporte essentiellement des noms duratifs, ce qui explique que dans puisse se voir substituer pendant ou au cours de dans 74,29 \% des énoncés.

En résumé, ces commutations montrent que dans est susceptible d'introduire un GP à sens causal, ce qui n'est pas enregistré dans les définitions lexicographiques en particulier (ainsi le Grand Larousse de la langue française (p. 1108) énumère-t-il « I. Marque le lieu [...], II. Marque le temps [...], III. Marque la manière d'être, l'état [...], IV. Marque l'approximation [...]). Le plus souvent suivi d'un nom prédicatif ${ }^{6}$, dans ne marque que la cause avec les noms [qualité] comme générosité ou bonté, mais associe la notion temporelle de «déroulement du procès » avec les noms [action], [événement] ou [état] tels ablation, choc ou abattement.

\subsection{Les noms et leur détermination}

On vient d'établir que suivant le type de nom qui est introduit par la préposition, les possibilités de commutations avec d'autres prépositions ou locutions prépositives se trouvent contraintes et n'apportent pas la même identité sémantique au GP : certains ne peuvent avoir qu'une interprétation causale (25,71\%), d'autres à la fois causale et temporelle $(74,29 \%$ ). Ce sont donc les différences distributionnelles qui permettent de distinguer les significations à attribuer au GP.

\subsection{1. les No (en position sujet)}

Dans la structure simple de type $N_{0} V\left(N_{1}\right)$ dans $N_{2}$, des manipulations montrent une relation forte entre le sujet $\mathrm{N}_{0}$ et le nom $\mathrm{N}_{2}$ du GP en dans, ce qui permet de conclure que, quoique le plus généralement supprimable et déplaçable en tête de phrase, le GP ne peut être considéré comme parfaitement autonome, "périphérique». Ainsi en (1), le possessif leur est obligatoire, donc également la co-référence du $\mathrm{N}_{2}$ avec le sujet Max et Léa - il en va de même dans les exemples (13) à (17). La relation peut être explicitée au moyen par exemple de Max et Léa ont de (la + une certaine) générosité, Max et Léa sont d'une certaine générosité. En (2) - mais aussi en (7) -, le possessif peut commuter avec le défini (qui est le déterminant actualisé en (4) ou (6) entre autres), mais dans inscrit le sujet dans l'état ou l'événement décrit par $\mathrm{N}_{2}$ : Max et Léa sont pris dans l'affolement (général) en (2), la moto est l'un des protagonistes du choc en (6), c'est le sujet qui fouille (et par là met au jour des ossements) en (8), qui gère des fonds de pension en (9), etc.

Cette inscription du sujet $\mathrm{N}_{0}$ dans ce que véhicule le complément $\mathrm{N}_{2}$ par le biais de la préposition parait caractériser dans (cf. Leeman 1997 opposant par exemple Le château a été détruit dans la bataille vs Le château a été détruit pendant la bataille) et être responsable de son aptitude à exprimer la relation causale. Cette implication « directe »

${ }^{6} \mathrm{~S}$. Hamon (2005) a montré que la relation causale dans $X$ cause $Y$ unit de manière privilégiée un $\mathrm{X}$ et un $\mathrm{Y}$ prédicatifs. 
(Danlos 1988) est confirmée par le fait que l'on ne peut opérer une séparation spatiale et/ou temporelle entre ce que décrit le GP et le reste de la phrase: *Elle est morte aujourd'bui dans l'attentat qui a eu lieu bier (vs Elle est morte aujourd'bui à cause de l'attentat qui a eu lieu bier), *Dans son altercation avec Lia à la maison, Max s'est mis à pleurer à l'école (vs Du fait de son altercation avec Lia à la maison, Max s'est mis à pleurer à l'école).

\subsubsection{Les $N_{1}$ (en position objet)}

Le GP causal introduit par dans concerne (au moins) trois paradigmes de noms : les noms de qualité de type vanité, les noms d'état ou de sentiment de type accablement, et les noms d'action ou d'événement de type ablation, tempête. Mais s'il y a plusieurs paradigmes de noms (ce que l'on peut démontrer par les différences distributionnelles instituées par la langue), alors doit-on considérer qu'il y a plusieurs types de complément ? L'analyse du corpus permet de progresser dans ce sens.

- lorsque le $\mathrm{N}_{1}$ concerne une seule personne (bien souvent le sujet animé de la phrase) et qu'il s'agit plutôt d'un comportement, d'une attitude de celle-ci (certes repérables à des manifestations concrètes) alors, dans ce cas-là, le GP à sens temporel reste diffus alors que la relation causale est claire (l'emploi du possessif s'avère acceptable) : Max dégoûte ses amis dans son abaissement devant ses supérieurs. Dans ce type d'emploi, uniquement causal, dans est associé à un nom de qualité tel que vanité, qui désigne une propriété permanente, ou à des états ponctuels :

(23) Dans sa vanité, Léa se croyait la plus belle.

(24) Dans sa joie, Léa faillit s'étrangler.

(25) Dans leur générosité, Max et Léa ont laissé tout l'béritage à Tom.

- lorsque les $\mathrm{N}_{1}$ renvoient à des lieux, l'interprétation causale diffère de la précédente car ce qui cause est extérieur aux $\mathrm{N}_{0}$ (on constate d'ailleurs que l'emploi du possessif est alors exclu) alors que précédemment la cause était une propriété des $\mathrm{N}_{0}$. Si dans les premiers énoncés dans établissait une coïncidence étroite entre le sujet et le GP, dans ces derniers la coïncidence véhiculée par dans s'opère davantage entre le procès qui inclut nécessairement le sujet (croupir, disparaitre...) et le GP :

(26) L'épave croupit lentement dans les eaux sales d'un quai désert.

(Le croupissement de l'épave est localisé spatialement et causalement à la fois par les eaux sales d'un quai désert.)

(27) La ville disparaît dans l'épaisseur du bocage de la forêt.

(La disparition de la ville a lieu en un certain endroit et du fait de l'identité de ce dernier d'épais bocage.)

- lorsque le $\mathrm{N}_{1}$ désigne un état (un sentiment), une action, un événement transitoire, alors le GP inclut de la cause et du temps : dire dans son accablement, c'est entendre "parce qu'il est accablé » et «durant le temps qu'il est accablé ». Comme précédemment, on constate que cette valeur «cause-durée » dénotée par le GP en dans ne s'opère pas de la même façon dans tous les énoncés. 
(i) Soit le $\mathrm{N}_{1}$ relie deux entités extérieures l'une à l'autre par le biais d'une action concrète. Dans ce type d'emploi l'emploi du possessif et de l'indéfini est bien souvent peu acceptable. On peut le constater dans Le château a été détruit dans la bataille, où être détruit est un fait concret qui touche sensiblement 'château' par le biais d'une action qu'est 'bataille', et on ne dirait pas ?? Le château a été détruit dans sa bataille.

(28) Max s'est coincé la main dans l'abaissement des stores.

(29) Max est mort dans l'abattage des arbres.

(30) Elle est morte dans l'accident.

(31) Dans l'explosion, des toits ont été arrachés.

(ii) Soit le $\mathrm{N}_{1}$ contient l'événement de la proposition ou le sujet de celle-ci, c'est-à-dire que bien souvent l'événement ou le sujet sont inhérents à l'action dénotée par le $\mathrm{N}_{1}$. Dans ces cas-là le possessif et le défini sont acceptables ${ }^{7}$. L'action est perçue dans son déroulement : ainsi Dans l'ablation d'un sein suppose que la modification fait en quelque sorte partie de la définition même de l'ablation.

(32) Dans l'ablation d'un sein, la plastique de la femme est modifiée.

(33) Dans son altercation avec Lia, Max s'est mis à pleurer.

(34) Il se découvre dans ses erreurs.

(35) Dans sa précipitation, Emma a perdu sa chaussure.

En conclusion, si les commutations (1.1) permettent de localiser un emploi causal pour dans, l'étude des classes lexicales en jeu (1.2) conduit à spécifier l'identité de cette préposition dans l'expression de cette relation. Cette spécificité est dans la nécessaire concomitance, coexistence en un même lieu et en un même instant, de ce que rapporte la phrase d'un côté et le GP en dans de l'autre. Nous rejoignons et confirmons ainsi l'hypothèse avancée indépendamment (Vaguer 2004) que le trait typique du signifié de cette préposition est à voir dans la notion de "coïncidence » (susceptible de se décliner en 'coïncidence totale' ou 'coïncidence partielle' selon les emplois, qu'ils soient spatiaux, temporels, causaux ou autres).

Ces observations conduisent en retour à affiner la perception sémantique intuitive première que l'on peut avoir d'énoncés tels que (36) ou (37), où la tradition verrait simplement un complément circonstanciel de lieu et l'analyse contemporaine un ajout locatif.

Si l'on prend l'exemple (36), on peut considérer que (on dit que) la batte croupissait parce qu'elle se trouvait dans le cabinet de toilettes, ou, en (37) que c'est parce que l'assassin est en prison (que l'on peut dire) qu'il croupit. Le verbe croupir

\footnotetext{
${ }^{7}$ Nous rejoignons sur ce point D. Leeman (1985: 99) qui notait l'importance de la présence du possessif et de son rôle dans les relations établies au sein de la phrase : «La relation de coréférence entre le possessif et le sujet de la phrase est nécessaire [...et] illustre la relation de cause à effet interne [dans mon affolement, je ne retrouvai plus ma voiture] alors que dans son abaissement a en quelque sorte le statut d'une circonstance extérieure dans Dans son abaissement, Paul fait peine à voir car s'il y a bien, en même temps qu'une notion de temps ('pendant qu'il est abaissé'), une relation de cause à effet ('Paul fait peine à voir parce qu'il est abaissé') c'est celui qui parle qui l'établit, elle n'est pas interne en ce sens qu'il ne s'agit pas de la cause de l'action du sujet mais de celle du jugement que l'on porte sur le sujet ».
} 
comporte par lui-même l'idée d'une immobilité contrainte (anormale) dont l'incidence est négative sur le sujet: la place d'une batte n'est pas le cabinet de toilettes, les prisonniers se morfondent dans leurs geôles.

(36) La batte de hockey croupissait dans notre cabinet de toilettes.

(37) Celui qui va vous tuer croupira dans une prison.

Si, dans Elle n'avait cessé de s'abêtir dans une profonde absence, le GP peut être décrit comme le lieu (l'état) dans lequel elle s'abêtit, «la profonde absence » a aussi un sens causal (elle s'abêtit parce qu'elle s'absente de tout, qu'elle ne s'intéresse à rien) ; mais en même temps, peut-être qu'il y a aussi une valeur conséquentielle : «à force de ne cesser de s'abêtir, elle tombe dans une profonde absence » (i.e. elle devient indifférente à tout). Cette profonde absence (aux choses) est le contexte où se produit et qui en même temps produit son abêtissement et est également un état nouveau pour elle qui en retour finit par la transformer (l'abêtir).

\section{L'identité propre de dans causal}

Comme on l'a vu, à ce sens causal peut être associée, dans certains énoncés, une valeur temporelle telle qu'illustrée par l'exemple (38b). Autrement dit, le dans causal inclut au cours de qui permet de mettre en évidence ce sens "cause-durée » (évoqué à plusieurs reprises par D. Leeman dans les années quatre-vingt): en somme, dans montre la profonde absence en train de se dérouler (et modifiant quelque peu l'attitude de elle en l'abêtissant). Ainsi dans doit être associé à un nom d'état ou incluant l'idée d'un processus en cours, d'un déroulement : découvrir dans la fouille, c'est découvrir dans le même temps qu'a lieu la fouille (ce qui n'est pas le cas avec à cause de, grâce à, à cause $d e$, du fait de qui ont uniquement un sens causal ${ }^{9}-i$.e. on peut très bien avoir un sens causal sans que soit associé à celui-ci une valeur temporelle - l'implication d'un sens temporel caractérise dans par rapport à ces prépositions). L'action introduite par dans ne peut pas avoir de conséquences dans le futur (38c) mais seulement au moment même où se déroule l'action ${ }^{10}$ : dans attribue donc la conséquence de l'action au déroulement même de l'action. Tout se passe dans les limites mêmes de l'action ${ }^{11}$. On

${ }^{8}$ J. Fodor (1970), A. Wierzbicka (1980) et J. St-Germain (1995) ne parlent pas de cause-durée mais d'unité de temps entre le fait-cause et le fait-effet (cf. Iordanskaja \& Arbatchewsky-Jumarie, 2000 : 124).

${ }^{9}$ De fait, dans ne peut introduire des noms de personnes ou d'objets (donc d'entités, par opposition aux noms prédicatifs) par opposition à ces locutions: (A cause de $+{ }^{*}$ Dans) Max, des tuiles ont été cassées, (Du fait de $+{ }^{*}$ Dans) la bombe, des vitres ont été brisées...

10 Alors que, on l'a signalé plus haut, une distance temporelle est possible, entre la cause et son effet, avec à cause de, grâce à, du fait de mais non avec dans :

(a) (A cause de + ?? Dans) son altercation avec Paul bier, Max est resté sombre aujourd'bui toute la journée.

(b) (Du fait de $+{ }^{*}$ Dans) cet accident, elle est morte six mois après.

(c) (Grâce à +*Dans) ses prières de la veille, le prêtre se sentait plus calme ce jour-là.

11 De même (la distance géographique impliquant du même coup une séparation des événements dans le temps), Elle est morte dans l'attentat de Port-Royal suppose qu'elle est morte à (la station) 
retrouve donc ici le statut «positionnel» (Laur 1993) et de «coïncidence interne» (Vaguer 2004) qui définissent dans.

(38) a. Elle n'avait cessé de s'abêtir dans une profonde absence.

b. Elle s'abêtit (dans + à cause de + au cours de) une profonde absence.

c. *Elle s'abêtit dans une profonde absence de la veille.

Par opposition à pendant ou au cours de qui montrent l'action dans son accomplissement, dans peut en inclure le terme, ce qui paraît être responsable du sens causal qui caractérise dans par rapport à ses concurrentes; en effet, dire (38a) Elle n'avait cessé de s'abêtir dans une profonde absence, c'est en même temps constater l'abêtissement provoqué par cette profonde absence et l'attribuer à son déroulement désigné, au temps écoulé qu'elle suppose. L'accomplissement de l'abêtissement est concomitant à celui de l'absence, alors que dire (38b) Elle s'abêtit (au cours d' + pendant) une profonde absence, c'est seulement localiser l'abêtissement d'Emma à l'intérieur de sa période d'absence. Ainsi on peut dire que pendant $N$ et au cours de $N$ introduisent un simple repère temporel alors que dans marque une coïncidence interne entre le procès et l'action dénotée par le $\mathrm{N}_{2}$. Il en va de même pour :

(39) Je m'embrouille (dans + à cause de + au cours de) mes explications.

Dire Je m'embrouille au cours de mes explications, c'est simplement situer le moment de la confusion (laquelle ne concerne pas nécessairement les explications elles-mêmes : Je m'embrouille (dans les dates) au cours de mes explications), et dire Je m'embrouille à cause de mes explications, c'est de même n'expliciter que le motif de la confusion (Je m'embronille (pendant l'exposé) à cause de mes explications), tandis que Je m'embrouille dans mes explications suppose que les explications elles-mêmes sont confuses (elles m'embrouillent).

Si cette hypothèse se révèle juste, cela signifierait que, d'une certaine manière, dans Il a été malade dans la nuit, il y a une relation entre la nuit et son indisposition, alors que dans Il a été malade pendant la nuit, on n'a qu'un simple repérage temporel - ce dont un prochain travail fera la vérification dans des corpus attestés.

\section{Conclusion}

L'identité propre de la préposition dans est de marquer la « coïncidence » (soit entre le procès énoncé dans la phrase et le GP, soit entre le $\mathrm{N}_{0}$ et le GP), qui peut être causale certes mais aussi temporelle et/ou spatiale. Cette hypothèse de définition a pu être mise en évidence à partir des sélections distributionnelles qui s'opèrent entre la préposition et le $\mathrm{N}_{2}$, en relation avec le reste de l'énoncé. Dans établit une relation de consubstantialité entre le sujet et son état; c'est ce qui explique les contraintes distributionnelles mais aussi la relation de dépendance instituée par dans: ainsi Dans l'ablation d'un sein, la plastique de la femme est modifiée suppose une coïncidence entre l'ablation et la modification du point de vue aspectuel, ce qui n'est pas le cas avec pendant, au cours $d e . .$. avec lesquels le sens est plutôt que la modification prend place au

Port-Royal, et l'on ne dirait pas : *Elle est morte à l'hôpital Saint-Michel dans l'attentat de Port-Royal (mais à cause de ou du fait de seraient possibles). 
cours de 'l'ablation'. De même, Dans sa colère, il jeta le vase par terre suppose que le sujet est en colère lorsqu'il agit : il y a une coïncidence du sujet et de l'état de colère (dans sa colère $=$ au cours de + à cause de), et dans Trois personnes sont mortes dans l'accident présente le décès comme concomitant à l'accident. Comme D. Leeman (1997) et C. Vandeloise (1999), nous avons pu montrer que l'usage temporel de la préposition dans n'est pas limité à l'expression du temps mais qu'il peut être associé à l'expression de la causalité, voire de la conséquence.

\section{[cause]}

\begin{tabular}{l}
\hline coïncidence entre le $\mathrm{N}_{0}$ et le GP \\
$-\mathrm{N}_{\text {qualité }}$ (propriété permanente), $\mathrm{N}_{\text {état }}$ \\
- Déterminant possessif
\end{tabular}

coïncidence entre le procès et le GP

$-\mathrm{N}_{\text {lieu }}$

- Déterminant possessif impossible

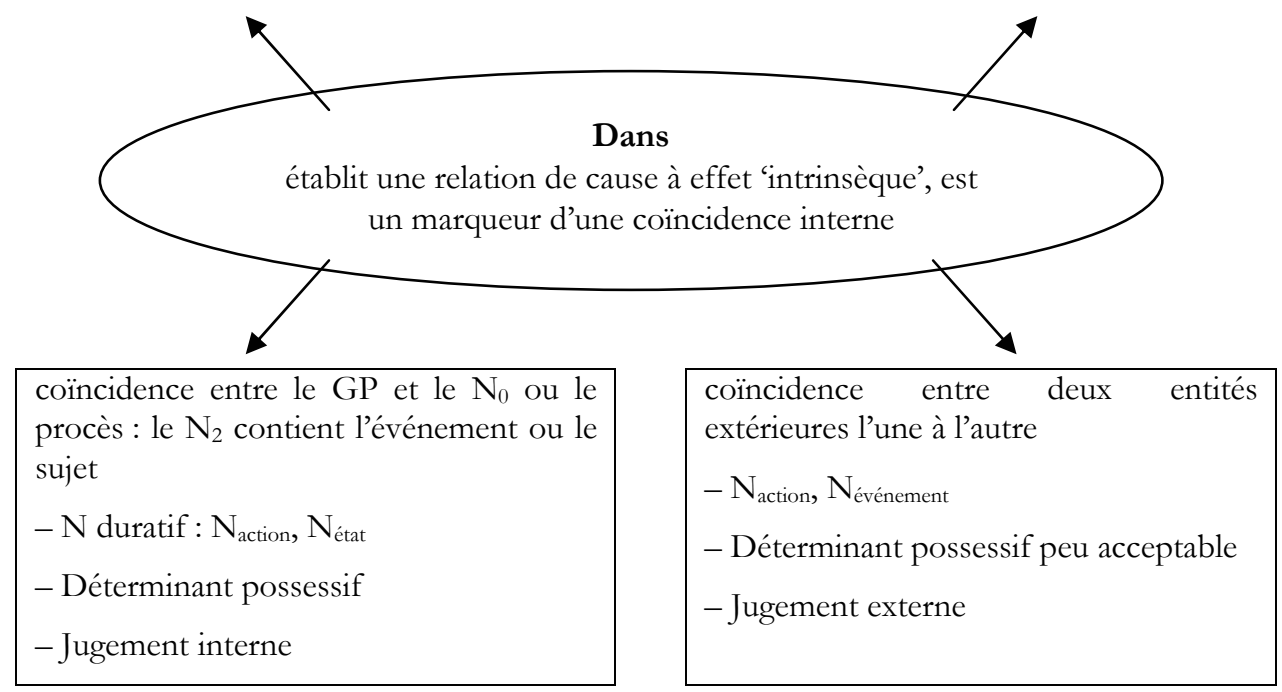

[cause-durée] 


\section{RÉFÉRENCES BIBLIOGRAPHIQUES}

Anscombre, J.-C., 1984, «La représentation de la notion de cause en langue », Cabiers de grammaire, $8: 5-53$.

Anscombre, J.-C., 1995, « Morphologie et représentation événementielle : le cas des noms de sentiment et d'attitude », Langue française, $105:$ 40-54.

BRøndal, V., 1950, Théorie des prépositions. Introduction à une sémantique rationnelle, Copenhague, Ejnar Munksgaard.

DANLOS, L., 1988, « Connecteurs et relations causales », Langue française, 77 : 92-127.

Flaux, N. \& VAN DE Velde, D., 2000, Les noms en français : esquisse de classement, Gap/Paris, Ophrys, coll. « L'essentiel».

FODOR, J., 1970, « Three Reasons for not Deriving kill from cause to die », Linguistic Inquiry, 1-4 : 429-438.

Groupe $\lambda-1,1975$, « Car, parce que, puisque », Revue romane, X-2 : 248-280.

Guilbert, L., Lagane, R. \& Niobey, G., 1971, Grand Larousse de la langue française en six volumes, Paris, Larousse.

Guillaume, G., 1919 in 1975, Le problème de l'article et sa solution dans la langue française, Paris/Québec, Nizet/Les Presses de l’Université Laval.

HAMON, S., 2005, La phrase double causale: propriétés syntaxiques et interprétations sémantiques, Thèse de doctorat, Université Paris X-Nanterre.

IORDANSKAJA, L. \& ARbATCHEWSKY-JUmARIE, N., 2000, «Quatre prépositions causales du français. Leur sémantisme et cooccurrence », Lingvisticae Investigationes, 23-1 : 115-159.

Lakoff, G., 1987, Women, Fire and Dangerous Things - What Categories Reveal about the Mind, Chicago/London, The University of Chicago Press.

LAUR, D., 1993, «La relation entre le verbe et la préposition dans la sémantique du déplacement », Langages, $110:$ 47-67.

LEEMAn, D., 1985, «Tentative de caractérisation d'un complément circonstanciel : Dans mon affolement, je lâchai mon panier de cerises », Linx, 12, 97-145.

LEEMAN, D., 1985/1986, "A propos du complément circonstanciel », Travaux de linguistique, $12 / 13: 177-193$.

LEEMAN, D., 1987, «Identification d'un complément en pour à sens de conséquence », Linx, $16: 120-144$.

LEEMAN, D., 1991, "Hurler de rage, rayonner de bonheur: remarques sur une construction en de», Langue française, 91, 80-101.

LEEMAN, D., 1994, "Remarques sur puisque et car», Théories, données et pratiques en français langue étrangère, Lille, Presses Universitaires de Lille : 113-128. 
Céline Vaguer

LeEman, D., 1995, «Pourquoi peut-on dire Max est en colère mais non *Max est en peur? Hypothèses sur la construction être en $N »$, Langue française, 105 : 55-69.

LEEMAN, D., 1997, «Contribution à l'élaboration du signifié de la préposition dans (dans et les noms d'action)", Actes du Sème colloque international de psychomécanique du langage (Seyssel) publié en 2001, Paris, Champion : 103-113.

LEEMAN, D., 1999, «Dans un juron, il sauta sur ses pistolets: aspects de la polysémie de la préposition dans », Revue de Sémantique et Pragmatique, 6 : 71-88.

LEEMAN, D., 1999, « La préposition : un 'auxiliaire' du nom ? », Langages, 135 : 75-86.

Moeschler, J., 1987, "Trois emplois de parce que en conversation », Cabiers de linguistique française, 12, 7-30.

NaZArenko, A., 2000, La cause et son expression en français, Paris, Ophrys.

PotTiER, B., 1955 in 1962, Systématique des éléments de relation. Étude de morphosyntaxe structurale romane, Paris, Klincksieck.

ST-GERMAIN, J., 1995, Incidence de la structure sémantique et communicative sur la structure syntaxique profonde des énoncés causatifs du français contemporain, Thèse de doctorat, Université de Montréal.

VAguer, C., 1998, Étude d'un complément circonstanciel: "Dans + Nom d'action », Mémoire de Maîtrise, Université Paris X-Nanterre.

VAguer, C., 2004, Les constructions verbales " $V$ dans GN". Approches syntaxique, lexicale et sémantique, Thèse de doctorat, Université Paris X - Nanterre.

VAGUER, C. (soumis) «Dans : l'expression de la coïncidence spatio-temporelle ».

VANDEloise, C., 1986, L'espace en français, Paris, Éditions du Seuil.

VAndeloise, C., 1999 «Quand dans quitte l'espace pour le temps», Revue de Sémantique et Pragmatique, 6, 145-162.

WierzBickA, A., 1980, Lingua mentalis, Sydney et al., Academic Press. 